\title{
Improving a Cluster Based Directional Channel Model in Realistic Macro-Cell Environment
}

\author{
Pedro Vieira • Paula Queluz • António Rodrigues
}

Published online: 31 March 2009

C) Springer Science+Business Media, LLC. 2009

\begin{abstract}
In this paper a realistic directional channel model that is an extension of the COST 273 channel model is presented. The model uses a cluster of scatterers and visibility region generation based strategy with increased realism, due to the introduction of terrain and clutter information. New approaches for path-loss prediction and line of sight modeling are considered, affecting the cluster path gain model implementation. The new model was implemented using terrain, clutter, street and user mobility information for the city of Lisbon, Portugal. Some of the model's outputs are presented, mainly path loss and small/large-scale fading statistics.
\end{abstract}

Keywords Wireless communications · Directional channel model $\cdot$ Clustering

\section{Introduction}

For the design and performance evaluation of mobile radio systems, realistic channel models are an absolute prerequisite. As systems become more elaborated, the requirements for channel models also change. For first- and second-generation systems, the Okumura-Hata model [1] and the wideband GSM channel model derived by the European research initiative COST 207 [2] have been sufficient. However, these models cannot be confidently applied to third- and fourth-generation systems.

\footnotetext{
P. Vieira $(\varangle)$

Department of Electrical Engineering, Lisbon Polytechnic Institute (ISEL), Lisbon, Portugal e-mail: pvieira@deetc.isel.ipl.pt

P. Vieira · P. Queluz · A. Rodrigues

Instituto de Telecomunicações/IST, Technical University of Lisbon, Lisbon, Portugal

P. Queluz

e-mail: paula.queluz@1x.it.pt

A. Rodrigues

e-mail: antonio.rodrigues@1x.it.pt
} 
A major shortcoming of the older, narrowband models is in the treatment of clustering of scatterers. In any wireless channel, scatterers are not distributed uniformly throughout the whole coverage area, but rather occur in clusters. One cluster of scatterers is always located around the Mobile Station (MS). Other clusters (henceforth called far clusters) are originated by high-rise buildings in urban environments, and hills/mountains in rural environments.

In recent years, new models were introduced that allow a realistic simulation of cluster properties. Two of the major attempts are the European Cooperation in the field of Scientific and Technical research (COST) 259 Directional Channel Model (DCM) [3], and its successor, the COST 273 Multiple Input Multiple Output (MIMO) channel model [4], for thirdand fourth-generation systems.

Cluster simulation is inevitably connected to Radio Environment(RE) description, mainly terrain height and clutter. The clusters and correspondent visibility region locations are directly associated with this information. Consequently, we present in this paper an extension of COST 273 channel model, introducing terrain and clutter information into radio channel generation. This attempt pretends to increase realism in radio channel cluster study keeping the COST 273 strategy, namely calibrated parameterization and execution speed.

This contribution increases realism and accuracy in the simulated channel behavior using improved algorithms for Line of Sight (LoS) modeling and path loss prediction, that directly affect the implemented cluster path gain model.

The paper is organized as follows. Section 2 introduces the new proposed model, i.e., clustering over irregular urban terrain. The new approach is presented and the cluster and visibility region generating Probability Density Function (PDF)s are derived. In the following, simulation results are presented using the proposed model in a Geographic Information System (GIS) tool for the city of Lisbon, Portugal.

Section 3 overviews the proposed approaches for path loss prediction and LoS modeling over irregular urban terrain. Section 4 introduces the considered cluster path gain model followed by the associated cluster power delay direction profile in Sect. 5. Fading is introduced in Sect.6. Section 7 presents a model implementation overview procedure, applied to the city of Lisbon, Portugal, followed by some of the model's outputs, mainly path loss and small/large-scale fading statistics. Finally, some comments conclude the paper.

\section{Clustering Over Irregular Urban Terrain}

\subsection{The Approach}

The proposed setup is presented in Figs. 1 and 2. A far cluster existence over irregular terrain is considered. The Base Station (BS) is installed above roof-top at height $h_{\mathrm{BS}}$ and is sup-

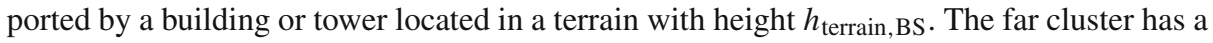
terrain height of $h_{\text {terrain,Cl}}$, a building height of $h_{\mathrm{B}, \mathrm{Cl}}$, and distance $r_{\mathrm{Cl}}$ to the BS. The visibility region distance to the far cluster is $d_{\mathrm{Cl}, \mathrm{vr}}$, the observation point height in the visibility region is $h_{\mathrm{MS} \text {,vr }}$, with a terrain height of $h_{\text {terrain, MS }}$. The observation point is surrounded with close buildings with height $h_{\mathrm{B}, \mathrm{vr}}$ and dists from the adjacent fatades by $w_{\mathrm{vr}} / 2$.

The far cluster and visibility region appearance depend on two paths, one between the BS and the far cluster and the other, between the far cluster and the visibility region.

The first path is inevitably associated to cluster generation, following the strategy of COST 273 channel model. It is proposed that cluster generation is dependent on the building height, referenced to the BS height. 


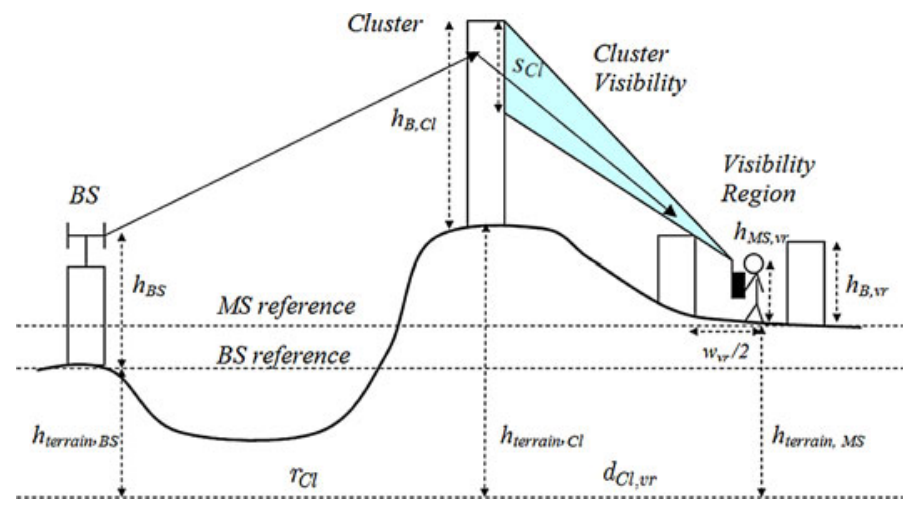

Fig. 1 Parameter definition for cluster and visibility region generation

Fig. 2 Azimuth and distances definition for cluster and visibility region setup
MS in Visibility Region

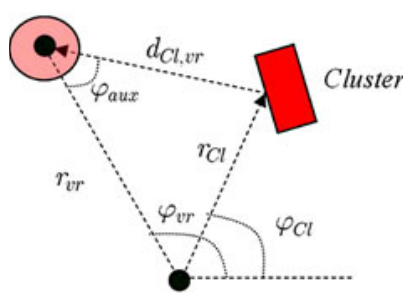

BS

The second path, between the cluster and the MS, is associated to the concept of visibility region. Each time a MS enters a visibility region, it is able to see the cluster location, so the corresponding cluster is made active.

Visibility is more probable if the BS, MS and far cluster are all aligned along a straight line, and becomes less likely in a broadside scenario. In [4] a solution using a 60 degree Gaussian distribution, was presented.

Considering Fig. 1, the far cluster will only be visible if it is higher than the visibility line height (in the clusters location) between the observation point and the cluster, passing tangent to the surrounding building. This means that the probability of existing a visibility region, will increase if the visibility region's close buildings are smaller or inexistent, if the street is wider, if the observation point is higher or if the associated far cluster is in the immediate surroundings of the MS.

Considering irregular terrain, the terrain height information is vital. The considered cluster height for the visibility region determination must be referred to the MS terrain height. So, from cluster to visibility region generation the reference height changes from the BS to the MS.

Figure 2 refers the main parameters used in the proposed approach in terms of cluster and visibility region positioning: $r_{\mathrm{Cl}}$ and $\varphi_{\mathrm{Cl}}$ are the cluster's distance and azimuth from the BS; $r_{\mathrm{vr}}$ and $\varphi_{\mathrm{vr}}$ are the visibility region's distance and azimuth; $d_{\mathrm{Cl}, \mathrm{vr}}$ is the distance from the cluster to the associated visibility region. Next section will present the PDF proposed for cluster and visibility region generation over irregular terrain. 


\subsection{Cluster Positioning}

Following the strategy of COST 273 channel model [4], the first step in the cluster positioning generation will be to define the number of far clusters. It depends on the simulation area and average number of clusters, which is specific for each RE.

The next step is to generate the clusters' positions. Considering the nomenclature already presented in Fig. 1, we propose a new generating PDF over irregular urban terrain. It follows COST 273 approach in using an exponential distribution based on the distance from the BS. The proposal is to add a component that includes building and height information. The generating PDF is,

$$
f_{\text {cluster }}\left(r_{\mathrm{Cl}}, \varphi_{\mathrm{Cl}}\right)=\frac{h_{\mathrm{Cl}}^{\mathrm{BS}}\left(r_{\mathrm{Cl}}, \varphi_{\mathrm{Cl}}\right)}{\overline{h_{\mathrm{Cl}}^{\mathrm{BS}}}} \cdot \frac{1}{2 \pi \sigma_{r}} \cdot \exp \left(-\frac{r_{\mathrm{Cl}}-r_{\mathrm{min}}}{\sigma_{r}}\right)
$$

where $h_{\mathrm{Cl}}^{\mathrm{BS}}$ is given by

$$
h_{\mathrm{Cl}}^{\mathrm{BS}}\left(r_{\mathrm{Cl}}, \varphi_{\mathrm{Cl}}\right)=h_{\mathrm{B}, \mathrm{Cl}}\left(r_{\mathrm{Cl}}, \varphi_{\mathrm{Cl}}\right)+\left(h_{\text {terrain, } \mathrm{Cl}}\left(r_{\mathrm{Cl}}, \varphi_{\mathrm{Cl}}\right)-h_{\text {terrain, } \mathrm{BS}}\right)
$$

and represents the overall cluster height referenced to the $\mathrm{BS}$ terrain height. The $\overline{h_{\mathrm{Cl}}^{\mathrm{BS}}}$ parameter is the average value of $h_{\mathrm{Cl}}^{\mathrm{BS}}\left(r_{\mathrm{Cl}}, \varphi_{\mathrm{Cl}}\right)$ over all the simulation area. $f_{\text {terrain }}$ is zero for $r_{\mathrm{Cl}}<r_{\min }$ or $h_{\mathrm{Cl}}^{\mathrm{BS}}<0 . r_{\min }$ and $\sigma_{r}$ are COST 273 structural parameters.

\subsection{Visibility Region Positioning}

The influence of irregular terrain and buildings is introduced in the following way. Consider $s_{\mathrm{Cl}}\left(r_{\mathrm{vr}}, \varphi_{\mathrm{vr}}\right)$ in Fig. 1 as the cluster's visibility extension from the visibility region observation point. This cluster's visibility extension can be interpreted as a measure of the visibility capability in the observation points, and used to model the visibility region generating PDF. It is given by,

$$
\begin{aligned}
& s_{\mathrm{Cl}}\left(r_{\mathrm{vr}}, \varphi_{\mathrm{vr}}\right)=\max \left(h_{\mathrm{Cl}}^{\mathrm{MS}}\left(r_{\mathrm{vr}}, \varphi_{\mathrm{vr}}\right)-h_{\mathrm{MS}, \mathrm{vr}}\left(r_{\mathrm{vr}}, \varphi_{\mathrm{vr}}\right)\right. \\
& \left.\quad-2 \frac{\left(h_{\mathrm{B}, \mathrm{vr}}\left(r_{\mathrm{vr}}, \varphi_{\mathrm{vr}}\right)-h_{\mathrm{MS}, \mathrm{vr}}\left(r_{\mathrm{vr}}, \varphi_{\mathrm{vr}}\right)\right)}{w_{\mathrm{vr}}\left(r_{\mathrm{vr}}, \varphi_{\mathrm{vr}}\right)} d_{\mathrm{Cl}, \mathrm{vr}}\left|\sin \left(\varphi_{\text {street }}\right)\right|, 0\right)
\end{aligned}
$$

where $r_{\mathrm{vr}}$ and $\varphi_{\mathrm{vr}}$ are the visibility region's distance and azimuth, respectively; $\varphi_{\text {street }}$ is the street orientation with respect to the cluster reflected path in the visibility region location and $w_{\mathrm{vr}}$ is the street width.

$h_{\mathrm{Cl}}^{\mathrm{MS}}$ is given by,

$$
h_{\mathrm{Cl}}^{\mathrm{MS}}\left(r_{\mathrm{vr}}, \varphi_{\mathrm{vr}}\right)=h_{\mathrm{B}, \mathrm{Cl}}+\left(h_{\text {terrain, } \mathrm{Cl}}-h_{\text {terrain, } \mathrm{MS}}\left(r_{\mathrm{vr}}, \varphi_{\mathrm{vr}}\right)\right)
$$

and represents the cluster height referenced to terrain height in the visibility region location.

After some trigonometrical manipulation it can be presented as a function of $r_{\mathrm{vr}}$ and $\varphi_{\mathrm{vr}}$ as:

$$
d_{\mathrm{Cl}, \mathrm{vr}}=\sqrt{r_{\mathrm{vr}}^{2}-2 r_{\mathrm{vr}} r_{\mathrm{Cl}} \cos \left(\varphi_{\mathrm{vr}}-\varphi_{\mathrm{Cl}}\right)+r_{\mathrm{Cl}}^{2}} .
$$

Hence, the new generating PDF over irregular terrain for the visibility regions is, 


$$
f_{\text {visibility }}\left(r_{\mathrm{vr}}, \varphi_{\mathrm{vr}}\right)=\frac{s_{\mathrm{Cl}}\left(r_{\mathrm{vr}}, \varphi_{\mathrm{vr}}\right)}{\overline{s_{\mathrm{Cl}}}} \cdot N\left(\varphi_{\mathrm{vr}}-\varphi_{\mathrm{Cl}}, \sigma_{\varphi_{\mathrm{Cl}}}\right)
$$

where $N\left(\varphi_{\mathrm{vr}}-\varphi_{\mathrm{Cl}}, \sigma_{\varphi_{\mathrm{Cl}}}\right)$ is a Gaussian PDF with average $\varphi_{\mathrm{vr}}-\varphi_{\mathrm{Cl}}$ and standard deviation $\sigma_{\varphi_{\mathrm{Cl}}}$.

Using this strategy it is possible to maintain backward compatibility with the measurement tuned COST 273 channel model, in terms of cluster and visibility region generation and, additionally, to consider influence of the terrain and clutter.

The proposed model was tested using a GIS tool previously built in MATLAB ${ }^{\circledR}$. The tool is based on $20 \times 20 \mathrm{~km}^{2}$ terrain/building database with a $20 \mathrm{~m}$ resolution, for the city of Lisbon, Portugal. So, in the presented figures, a pixel represents a $20 \mathrm{~m}$ square unit.

Briefly, the GIS tool is built using different layers. The BS layer is based on the real location of a 3G network, outdoor macro-cells only. Multiple sectorized sites are placed in the tool's Virtual Cell Deployment Area (VCDA) using parameterization like BS height, cell orientation, BS latitude and longitude, etc.

The considered MS layer is built using multiple vehicular MSs that travel around the VCDA using a realistic mobility model. The mobility model was previously tuned and validated with Lisbon vehicular traffic measurements [5] and [6]. The MSs move using a detailed vector street database for the city.

\subsection{Implementation of Cluster Generating PDF}

For each BS, an action region is built, that is basically a subgroup of the terrain database array centered in the MS's locations and with considerable size, allowing to detect even extremely far away clusters. The action region is implemented in order to increase the tool's efficiency and speed up the calculations. We assumed Generalized Bad Urban (GBU) environment for Lisbon (using COST 273 parametrization). We will now present the models's generating PDFs for a selected BS's vicinity.

Figure 3 presents the cluster generating PDF used in COST 273 considering distance dependency only. It is important to notice that Lisbon's terrain profile and building height variation are quite big. Additionally, due to cell planning and interference optimization issues, the BSs are normally placed in lower structures and near high-rise buildings, which will certainly work as clusters.

Figure 4 highlights building and terrain influence, since it presents $h_{\mathrm{Cl}}^{\mathrm{BS}} / \bar{h}_{\mathrm{Cl}}^{\mathrm{BS}}$ in the $\mathrm{BS}$ action region. Terrain variation and building height dispersion is easily noticed. The map presents the surrounding area of a BS located near Av. Brasil, in central Lisbon.

Figure 5 presents $f_{\text {cluster }}\left(r_{\mathrm{Cl}}, \varphi_{\mathrm{Cl}}\right)$, the cluster generating PDF, introduced in (1). Backward compatibility with COST 273 is a fact and additionally, buildings and terrain influence is introduced.

The work presented in this paper is simulation oriented. No experimental data was used for model calibration. For this reason, backward compatibility was tried with experimentally validated COST 273 channel model. The experimental validation of the presented model would be highly interesting for further work.

\subsection{Implementation of Visibility Region Generating PDF}

The number of visibility regions associated to each cluster is modeled as a Poisson-distributed random variable with mean value of two. We will now illustrate the models's generating PDF $\mathrm{s}$ for a selected BS's vicinity and for a previously generated cluster. 


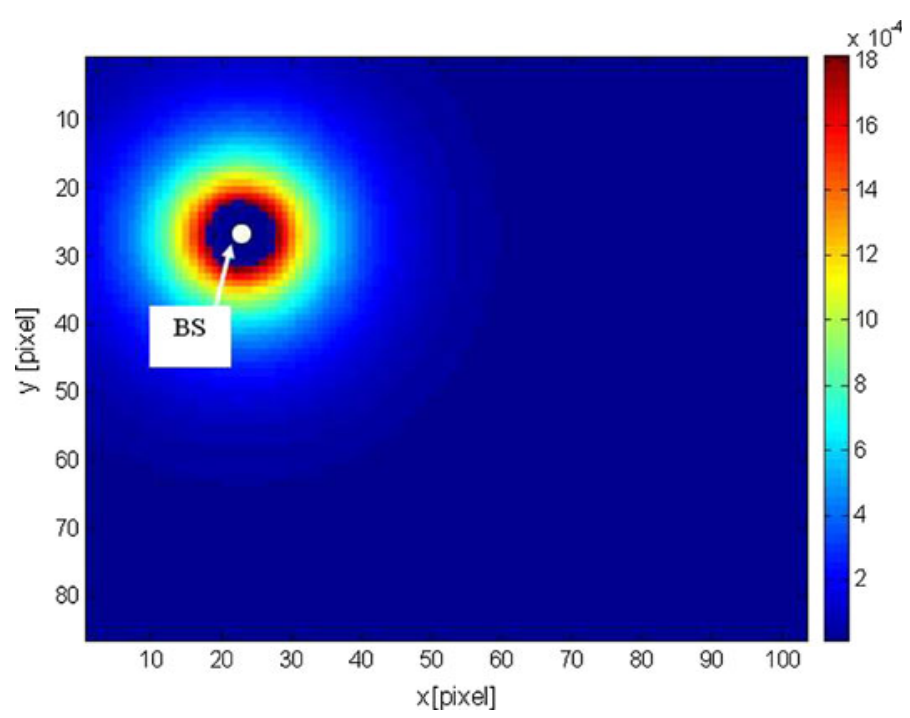

Fig. 3 Cluster generating PDF for COST 273 channel model

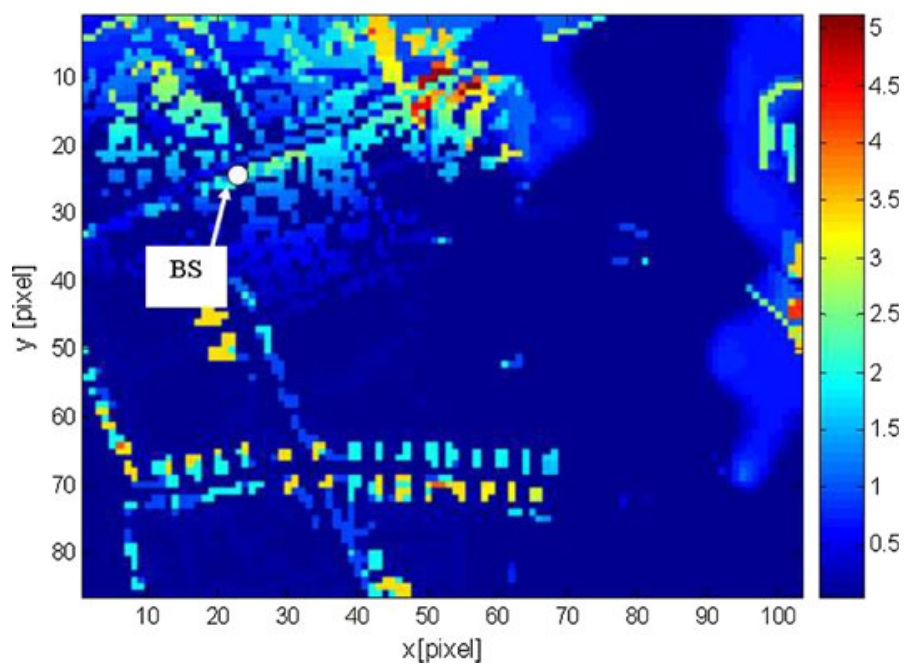

Fig. 4 Representation of $h_{\mathrm{Cl}}^{\mathrm{BS}} / \overline{h_{\mathrm{Cl}}^{\mathrm{BS}}}$ in the $\mathrm{BS}$ action region

Figure 6 presents the Gaussian visibility region generating PDF used in COST 273 channel model. Figure 7 shows the visibility function $s_{\mathrm{Cl}}\left(r_{\mathrm{vr}}, \varphi_{\mathrm{vr}}\right)$, for a specific cluster. The street orientation influence in cluster visibility is well noticed. We present in Fig. 8 the visibility region generating PDF, $f_{\text {visibility }}\left(r_{\mathrm{vr}}, \varphi_{\mathrm{vr}}\right)$. The azimuths close to clusters azimuth suffer an increased probability, and due to $s_{\mathrm{Cl}}$ influence, street orientation, clutter and terrain effect is added.

Next, the cluster's and visibility regions's positions are randomly determined using the presented two dimensional generating PDFs. Rejection method was applied in the $r_{\mathrm{Cl}}, \varphi_{\mathrm{Cl}}$ and $r_{\mathrm{vr}}, \varphi_{\mathrm{vr}}$ random number generation. 


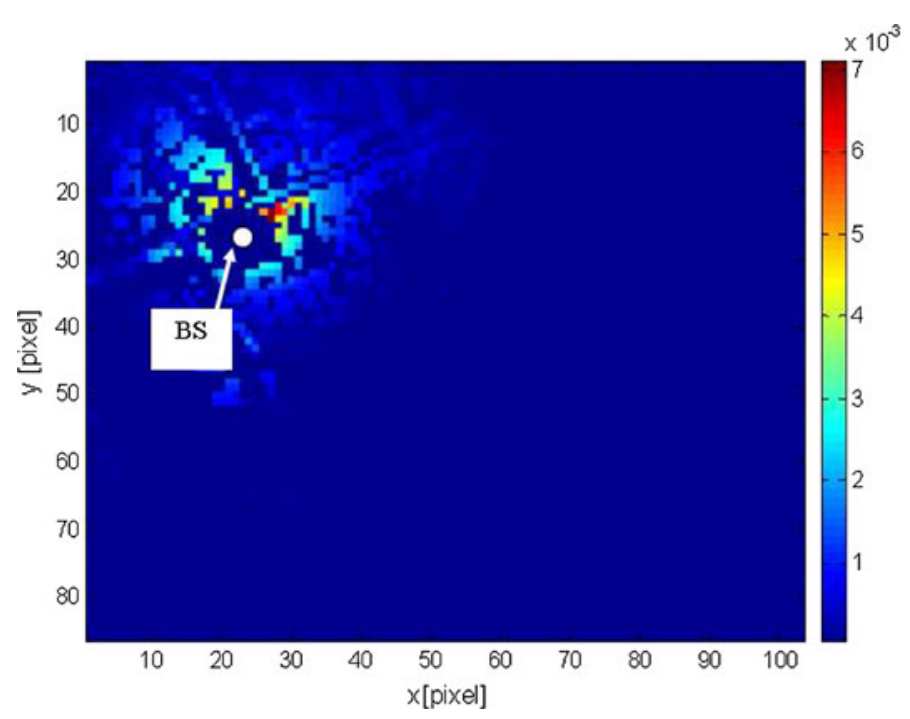

Fig. 5 Cluster generating PDF, $f_{\text {cluster }}\left(r_{\mathrm{Cl}}, \varphi_{\mathrm{Cl}}\right)$ considering terrain and buildings

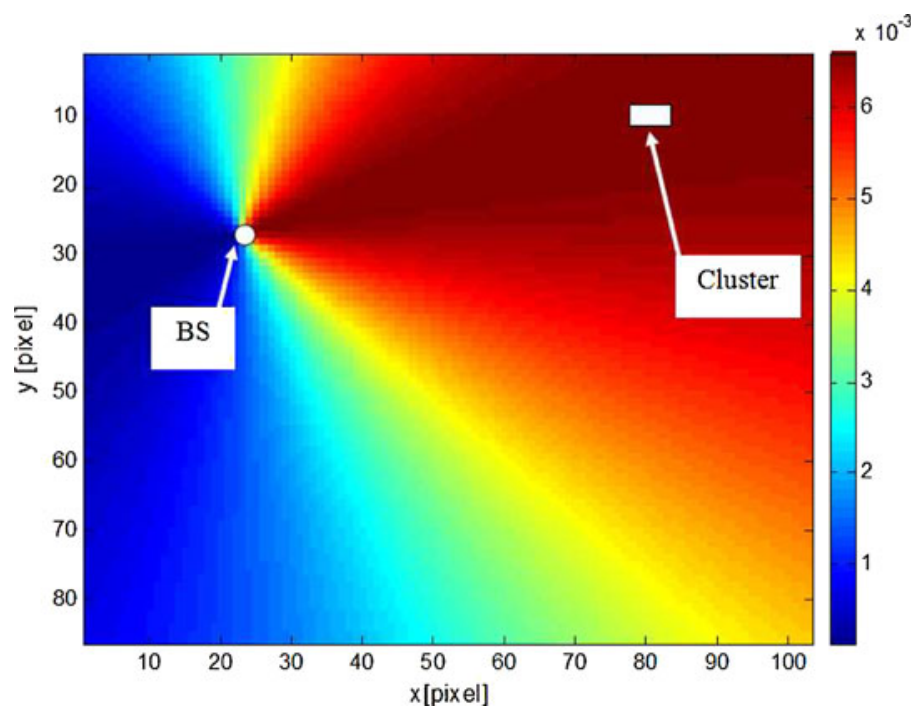

Fig. 6 Visibility region generating Gaussian PDF for COST 273 channel model

\section{Path Loss and Line-of-Sight Modeling}

\subsection{The Path Loss Model}

The implemented propagation model is the COST 231 Walfisch-Ikegami (WI) and the implementation area is Lisbon.

The model is chosen because it is the selected one for the new COST 273 [4] macro-cell framework. Additionally, Lisbon presents irregular terrain, which motivated us to develop 


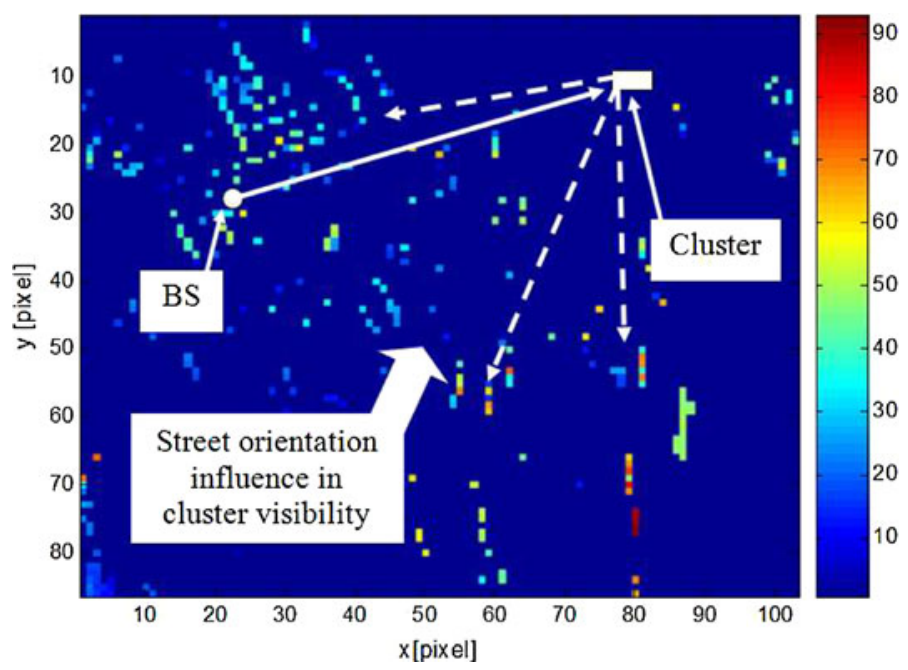

Fig. 7 Representation of $s_{\mathrm{Cl}}\left(r_{\mathrm{vr}}, \varphi_{\mathrm{vr}}\right)$ in the BS action region for a designed cluster [m]

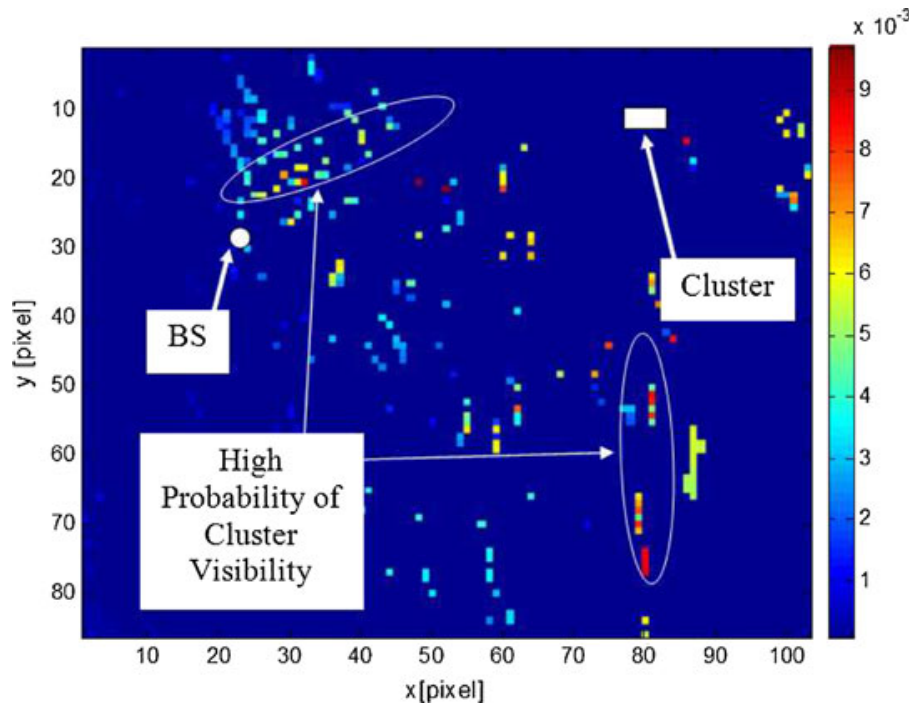

Fig. 8 Visibility region generating PDF, $f_{\mathrm{visibility}}\left(r_{\mathrm{vr}}, \varphi_{\mathrm{vr}}\right)$ considering terrain and buildings

a new algorithm that converts dynamically GIS terrain and clutter into the flat terrain input parameterization needed for COST 231 WI model.

Under this algorithm, a new method for average building height calculation is proposed, using Epstein-Peterson's multiple knife-edge diffraction theory [7].

\subsection{Line-of-Sight Modeling}

The LoS modeling is an important subject in what refers to signal propagation prediction. In recent COST 259 and COST 273 channel models, the associated probability of LoS existence 
Fig. 9 Definition of parameters used in COST 231 WI model and LoS determination strategy

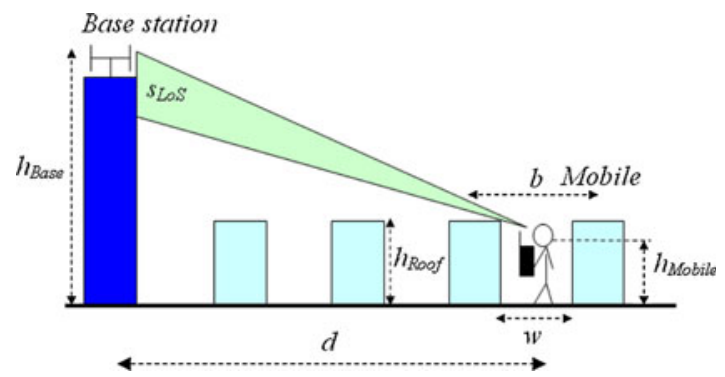

is given by,

$$
P_{\mathrm{LoS}}= \begin{cases}\frac{h_{\text {Base }}-h_{\text {Roof }}}{h_{\text {Base }}} \cdot \frac{d_{\mathrm{co}}-d}{d_{\mathrm{co}}}, & d<d_{\mathrm{co}} \wedge h_{\text {Base }}>h_{\text {Roof }} \\ 0, & d \geq d_{\mathrm{co}} \wedge h_{\text {Base }} \leq h_{\text {Roof }}\end{cases}
$$

where $d$ represents distance from BS and $d_{\text {co }}$ is a cut-off distance.

A new approach is introduced considering COST 231 WI model setup, and presented in Fig. 9. The BS is only visible from the MS location if its height $\left(h_{\text {Base }}\right)$ is higher than the visibility line height between the MS and the BS, passing tangent to the MS's adjacent building, with height $h_{\text {Roof }}$.

Additionally, and considering the street orientation effect, the most critical situation in terms of $\operatorname{LoS}$ existence will be for $\varphi=90$ or 270 , when the road orientation is perpendicular to the direct radio path. For these cases, we consider that the distance from the MS to the close buildings is $w / 2$. For $\varphi$ close to 0 or 180 , the LoS probability should increase, approaching 1. Hence, the proposed probability of LoS existence is given by,

$$
P_{\mathrm{LoS}}= \begin{cases}1-\frac{2\left(h_{\text {Roof }}-h_{\text {Mobile }}\right) d|\sin \varphi|}{w\left(h_{\text {Base }}-h_{\text {Mobile }}\right)}, & d<d_{\text {co }} \wedge s_{\text {LoS }} \geq 0 \\ 0, & d \geq d_{\mathrm{co}} \wedge s_{\mathrm{LoS}}<0\end{cases}
$$

with $s_{\text {LoS }}$ set as,

$$
s_{\text {LoS }}=h_{\text {Base }}-h_{\text {Mobile }}-\frac{2\left(h_{\text {Roof }}-h_{\text {Mobile }}\right)}{w} d|\sin \varphi|
$$

and drawn in Fig. 9.

The proposed approach introduces more realism in the LoS stochastic generation, considering COST 231 WI macro-cell model setup. The LoS existence is not only dependent on the BS-MS distance and BS to roof-top height. The MS' surrounding environment is also important and it must be considered.

Figure 10 presents the probability of LoS existence from COST 273 model [4] and described by Eq. 7. The BS location was randomly chosen in the already presented terrain plus clutter database. $h_{\text {Base }}$ and $h_{\text {Mobile }}$ are 30 and $1.5 \mathrm{~m}$, respectively. Figure 11 presents the new approach for LoS probability calculation using Eq. 8. The street width and orientation influence in the LoS modeling is introduced as well as the terrain and building heights variation.

\section{Cluster Path Gain Model}

On a channel having an equivalent impulse response with multiple clusters, whose time variations in amplitude are uncorrelated, the total path gain (i.e., the inverse of the path loss) $P$, 


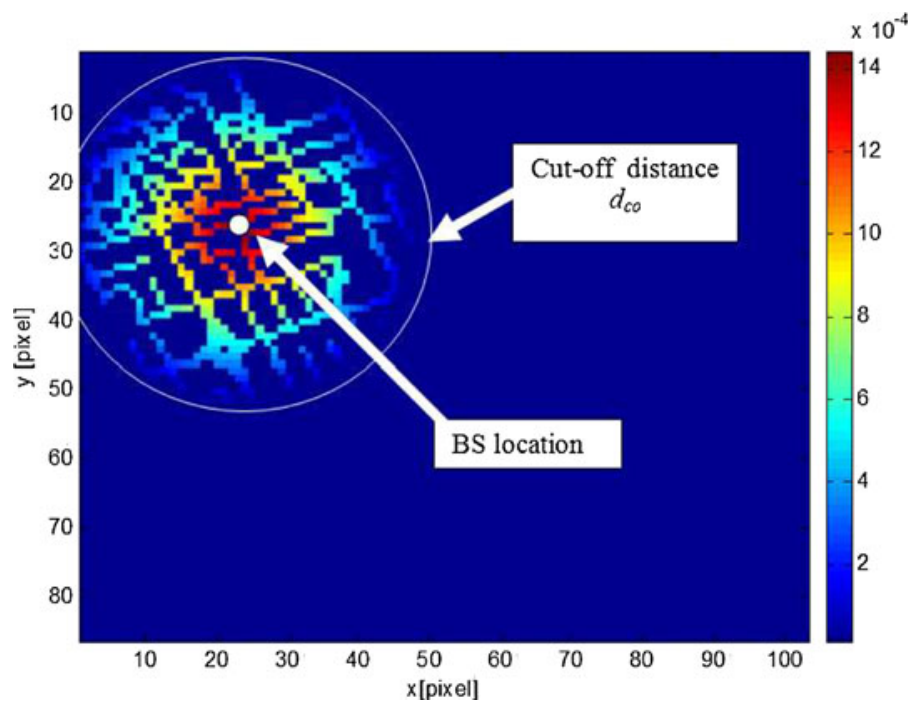

Fig. 10 Probability of LoS existence according to COST 273 model

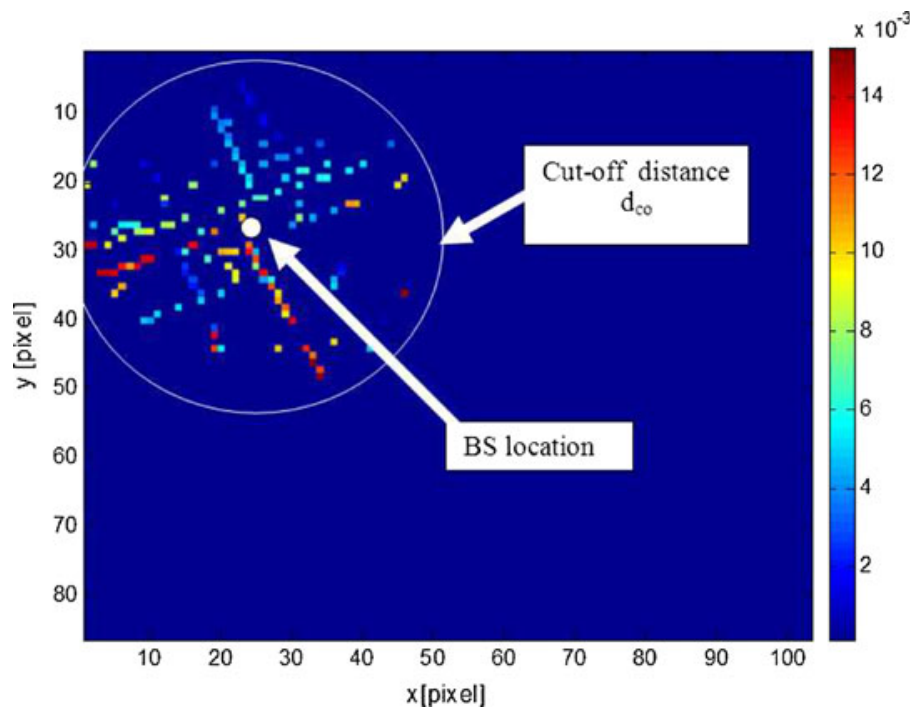

Fig. 11 Probability of LoS existence using the new approach

is composed of the sum of the cluster path gains:

$$
P=\sum_{m=1}^{M} P_{m}
$$

where $M$ is the total number of clusters. A practical approach [8] to model the cluster path gains is to determine a distance-dependent correction factor $s(d)$ that relates the expectation of $P_{m}$ to the expectation of the total path gain. The path gain of the first and always present cluster, $P_{1}$, is then simply modeled by the total path gain divided by $s(d)$; additional clusters 
have their path gains modeled by $P_{1}$ multiplied with a function that decreases in value with increasing excess delay, referred to the first cluster delay.

Taking all this information into account, the COST project developed a cluster path gain model which was tuned through comparison of resulting Root Mean Square (RMS) delay and angular spreads with those estimated from measured data. The model is formulated as [8],

$$
P_{m}= \begin{cases}S_{1} \frac{P_{\mathrm{NLOS}}}{S(d)}+A_{L}^{2}\left(\frac{\lambda}{4 \pi d}\right)^{2} & \text { for } m=1 \\ A_{m}^{2} S_{m} \frac{P_{\mathrm{NLOS}}}{s(d)} 10^{\frac{-k_{\tau} \min \left(\tau_{m}-\tau_{1}, \tau_{\mathrm{B}}\right)}{10}} & \text { for } m>1\end{cases}
$$

where $P_{\mathrm{NLOS}}$ represents the Non Line of Sight (NLOS) path gain; $A_{L}$ is a transition function for activating the LoS component; $S_{m}$ is the shadow fading gain which will be discussed in more detail in Sect. 6.1; $A_{m}$ is the transition function [4], for activating/deactivating a cluster. The parameters $k_{\tau}$ and $\tau_{\mathrm{B}}$ characterize the path gain conditioned on excess delay $\tau_{m}-\tau_{1}$.

An exponential decay is applied up to excess delay $\tau_{\mathrm{B}}$. Beyond this delay, the path gain is constant which results in the existence of some clusters with significant power at long delays. The LoS path gain is modeled by free space path gain multiplied by a transition function $A_{L}^{2}$. The transition function takes values between zero and one and is used for modeling transitions between LoS and NLoS [4].

To determine where there is LoS, a dynamic geometrical model with visibility areas very similar to the one discussed in [4] is proposed. To comply with the probability of LoS given by Eq. 8 , circular areas with radius $R_{L}$ are distributed with an area density $\rho_{L} \cdot \bar{p}_{\text {LoS }}$ is the average LoS probability is the BS/MS surrounding area and $L_{L}$ is a transition region. Hence,

$$
\rho_{L}=\frac{\bar{p}_{\mathrm{LoS}}}{\pi\left(R_{L}-L_{L}\right)^{2}}\left[m^{-2}\right] .
$$

When a mobile enters one of these circles the transition function, $A_{L}$ is not null and the LoS path gain is added (see Eq. 11).

When selecting appropriate parameter values, it is important to consider the influence of cluster path gains and cluster positions, in delay and angle, on the channel time- and angular dispersion. The suggested parameter values for the cluster path gain modeling are listed in [4].

\section{Cluster Power Delay Direction Profile}

The Power-Delay-Direction Profile (PDDP), for a cluster, reflects the average relative power as a function of relative delay and directions within a cluster, and is characterized by the PDDP function $P\left(\tau, \varphi, \varphi^{\prime}\right)$. $\varphi$ is the azimuth angle of incidence at the $\mathrm{BS}, \varphi^{\prime}$ is the azimuth angle of departure at the MS and $\tau$ is the delay. For simplicity reasons, no elevation angle is considered. It is proposed in [4] that the PDDP for each cluster should be represented as:

$$
P\left(\tau, \varphi, \varphi^{\prime}\right)=P_{\tau}(\tau) P_{\varphi}(\varphi) P_{\varphi^{\prime}}\left(\varphi^{\prime}, \tau\right)
$$

A benefit of the previous decomposition is that information is separately available in the literature for each of the profiles on the right-hand side of Eq. 13.

The Power-Delay-Direction Profile (PDDP), $P_{\tau}(\tau)$, has attracted the most attention due to its strong impact on inter-symbol interference, which requires the use of equalizers and multipath mitigation. An exponentially decaying PDP is usually accepted as a suitable model. The exponential profile is characterized by the decay constant $\sigma_{\tau}$, which is the well known 
RMS delay spread,

$$
P_{\tau}(\tau)= \begin{cases}\frac{1}{\sigma_{\tau}} e^{-\left(\tau-\tau_{m}\right) / \sigma_{\tau}}, & \tau \geq \tau_{m} \\ 0, & \text { otherwise }\end{cases}
$$

The azimuth spread at the BS was first studied indirectly using space diversity measurements [9] where a Gaussian profile was assumed. Later, Pedersen et al. [8] showed from direct measurements that a Laplacian function (15) was a better fit. The parameter $\sigma_{\varphi}$ that characterizes this function is referred to as the azimuth spread,

$$
P_{\varphi}(\varphi)=\frac{1}{\sigma_{\varphi} \sqrt{2}} e^{-\sqrt{2}\left|\varphi-\varphi_{m}\right| / \sigma_{\varphi}} .
$$

Incorporating these findings, COST 273 DCM presents the following structure for the angular profile at the MS: for the first cluster $(m=1)$, a uniform azimuth distribution is used for waves with relative delay less than $\tau_{C}$, and a combination of two Laplacian distributions is used when the cluster delay is beyond $\tau_{C}$,

$$
P_{\varphi^{\prime}}\left(\varphi^{\prime}, \tau\right)= \begin{cases}\frac{1}{2 \pi} & \text { for } \tau_{1}<\tau<\tau_{1}+\tau_{C} \\ \frac{1}{\sigma_{\varphi^{\prime}} 2 \sqrt{2}}\left(e^{-\frac{\sqrt{2}\left|\varphi^{\prime}-\varphi_{A}^{\prime}\right|}{\sigma_{\varphi^{\prime}}}}+e^{-\frac{\sqrt{2}\left|\varphi^{\prime}-\varphi_{B}^{\prime}\right|}{\sigma_{\varphi^{\prime}}}}\right) & \text { for } \tau \geq \tau_{1}+\tau_{C} .\end{cases}
$$

Here, $\sigma_{\varphi^{\prime}}$ is the azimuth spread and $\varphi_{A}^{\prime}$ and $\varphi_{B}^{\prime}$ are the directions of the street canyon in which the MS is located. These directions can be obtained directly from road orientation $\varphi_{R}$, i.e., by $\varphi_{A}^{\prime}=\varphi_{1}-\varphi_{R}$ and $\varphi_{B}^{\prime}=\varphi_{1}-\varphi_{R}+\pi$. Delayed clusters are characterized by a single Laplacian distribution around the direction $\varphi_{m}^{\prime}$ of the associated $m$ th cluster.

\section{Fading Statistics}

\subsection{Cluster Spreads and Large-Scale (Shadow) Fading}

The complete model for shadow fading of cluster envelopes and angular spreads at the BS is a variant of the model by Greenstein [10], and is formulated in terms of the cluster spreads $\sigma_{\tau, m}, \sigma_{\varphi, m}$ and the cluster shadow fading gain $S_{m}$, according to the following:

$$
\begin{aligned}
S_{m} & =10^{s_{s h f}} X_{m} / 10 \\
\sigma_{\varphi, m} & =m_{S \varphi} 10^{S_{s \varphi} Y_{m} / 10} \\
\sigma_{\tau, m} & =m_{S \tau}\left(\frac{d}{1000}\right)^{\varepsilon} 10^{s_{s \tau}} Z_{m} / 10
\end{aligned}
$$

where $X_{m}, Y_{m}, Z_{m}$ are random Gaussian variables with zero mean, unit variance, and crosscorrelations $\rho_{X Y}, \rho_{X Z}, \rho_{Y Z}$, respectively.

The random variables $X_{m}, Y_{m}, Z_{m}$ have exponential autocorrelation functions with autocorrelation lengths $L_{S}, L_{\tau}, L_{\varphi}$. The parameters $s_{s h f}, s_{s \tau}, s_{s \varphi}$ are standard deviations expressed in $\mathrm{dB}$. The median azimuth spread is $m_{s \varphi}$, while $m_{s \tau}$ is the median delay spread at a distance $d=1,000 \mathrm{~m}$ from the associated BS. The dimensionless exponent $\epsilon$ determines the distance dependence of the delay spread, as reported in [10]. 


\subsection{Small-Scale Fading}

The envelopes of narrowband signals received over fading radio channels are well-known to often vary with Rayleigh or sometimes Ricean distributions. In a directional wideband channel model the fading statistics in each resolvable delay/angle bin should be considered.

The extended COST 273 model therefore imposes Rayleigh fading in all resolvable delay/angle bins but with a strong, persistent Multi-Path Component (MPC) added to give Ricean fading in the first bin. Ricean distribution is often described in terms of Ricean $K_{0}$ factor, which is defined as the ratio between the power of the direct component and power of the Rayleigh part, i.e., the variance of the multipath.

For macrocells, in case there is LoS between the MS and the BS, the power of the deterministic quasi-LoS component is derived from the previous $K_{0}$ factor. Otherwise, if there is NLoS, $K_{0}$ is much lower. The Rice factor is modeled as log-normal distributed random variable, whose mean depends on the Excess Path Loss (EPL),

$$
K_{0}(\mathrm{EPL})=N\left(m_{K}, \sigma_{K}\right)=N\left(\frac{26-\mathrm{EPL}[\mathrm{dB}]}{6}, 6\right)[\mathrm{dB}] .
$$

The excess pathloss is the deviation (in $\mathrm{dB}$ ) between the narrowband free space pathloss and the current pathloss. $K_{0}$ is spatially generated using a log-normal distribution with mean $m_{K}$ dependent on the EPL, standard deviation $\sigma_{K}$ and an exponential autocorrelation function with correlation length $L_{K}$.

With the PDDPs, the positions, and the relative powers of the clusters set, all that remains is to simulate the MPC s that make up each cluster. According to [4], 20 MPCs are proposed for simulating each cluster, and this was the default value used in the model implementation, described in the next section.

\section{Model Implementation}

A macroscopic view of the developed simulator's structure is presented in Fig. 12. The channel model simulator was developed in MATLAB ${ }^{(}$and requires five input files: building data (raster), terrain data (raster), street data (vectors), BS and MS information.

The BS data stores the geographical positioning, height, power parameters, antenna configuration, radiation pattern, and system parameters like handover hysteresis and offset.

The MS positional data is generated using a new mobility model developed and validated [11] for Lisbon city. After each MS movement, all the MS parameterization is stored, mainly positioning, speed, direction of movement, power parameterization and system parameterization.

For each BS-MS radio-link, the new directional channel model is applied. The model's implementation proposed in this paper is a complex task compared to that of implementing simpler models, due to the combined modeling of small-scale and large-scale effects.

The MS local movement is compared with the size of the local area, which is defined as the area over which all large-scale fading parameters can be viewed as constants. The large-scale parameters are updated when the MS movement exceeds the size of the local area, which was set to $20 \lambda$. To this end, a large number of channel realizations were generated that simulate conditions at various distances between the MS and the BS, using an implementation of the proposed model. The tested urban RE is located in the city of Lisbon, Portugal, and is considered GBU. Several virtual test MSs travel around the city for periods of $10 \mathrm{~min}$. 


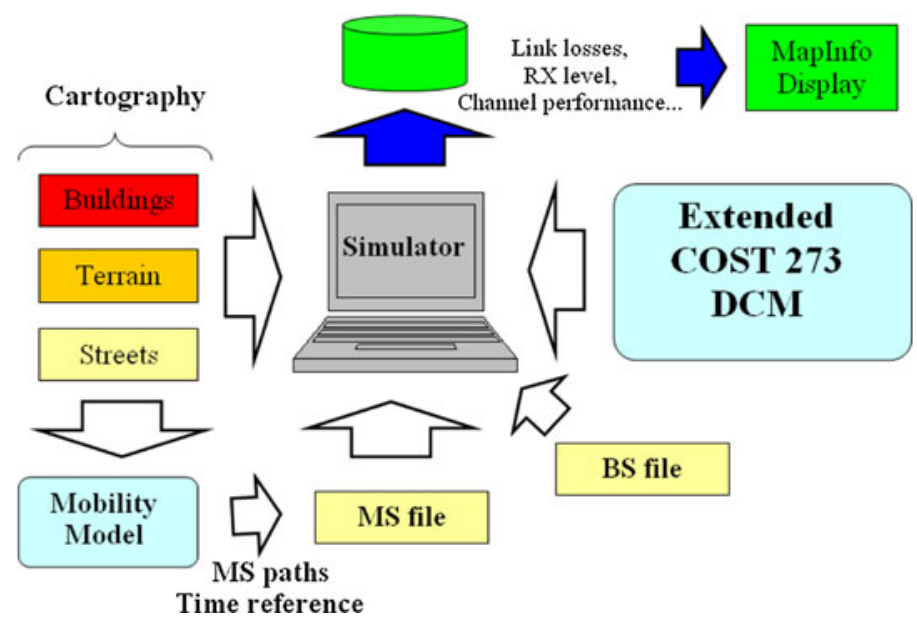

Fig. 12 Simulator's block diagram

The values for the needed external parameters are imported from the BS and MS setup files. We assume a macro-cell BS positioning and parametrization. A carrier frequency of $2 \mathrm{GHz}$ was assumed.

\subsection{Path Loss}

The radio link average path loss was obtained by averaging 50 realizations within a round local area with a diameter of $20 \lambda$.

Figure 13 presents the path loss for the Lisbon GBU radio environment as a function of the BS-MS distance for two virtual test mobiles. Using this and similar data, it was possible to calculate Lisbon's average path loss decay with distance, which rounds $30 \mathrm{~dB} /$ decade.

Other simulations have shown that the GBU path loss follows, on average, the COST 231 Walfisch-Ikegami model very well, except where the occurrences of LoS lead to lower path losses than those reflected by the COST 231-Walfisch-Ikegami model. This specially happens for distances less than $500 \mathrm{~m}$ from the BS [12].

\subsection{Small-Scale Fading}

Channel realizations for the Lisbon's GBU RE were used to determine small-scale fading statistics of simulation results. Figure 14 shows the average Rice $K_{0}$ factor as a function of BS-MS distance for two virtual test mobiles. $K_{0}$ was calculated after an averaging process using 50 channel realizations within a $20 \lambda$ local area $(3 \mathrm{~m})$. The fast fading reveals to be Rice distributed for shorter distances to the BS, as expected, due to the increased LoS probability. For distances farther away from the BS, fading tend to be Rayleigh distributed, with a $K_{0}$ factor closer to zero. 


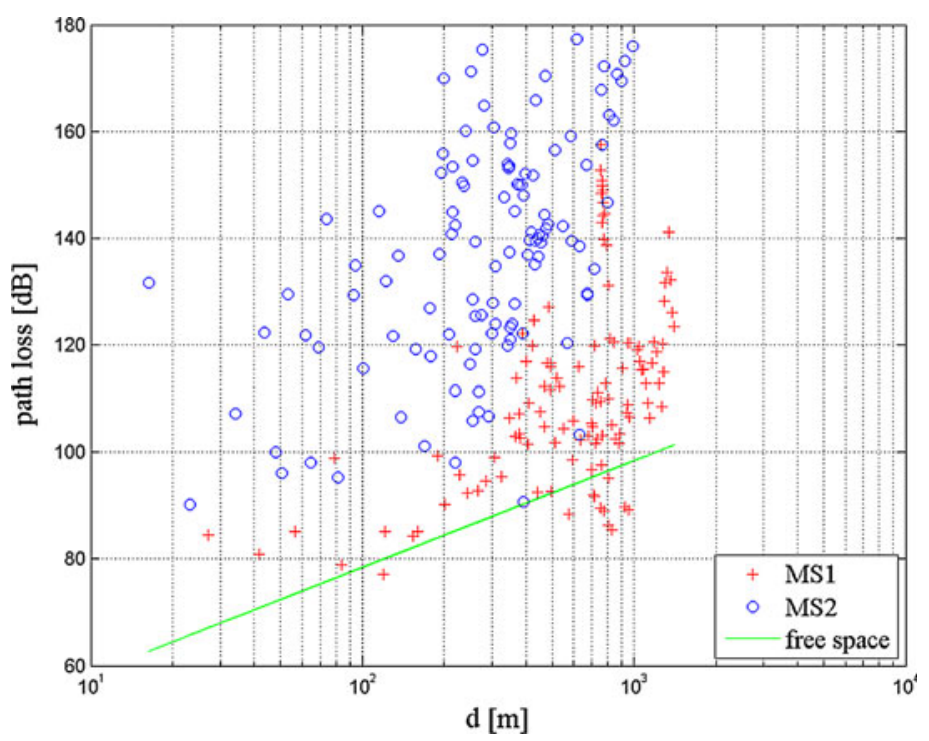

Fig. 13 Simulated channel path loss for two mobiles in the Lisbon GBU environment

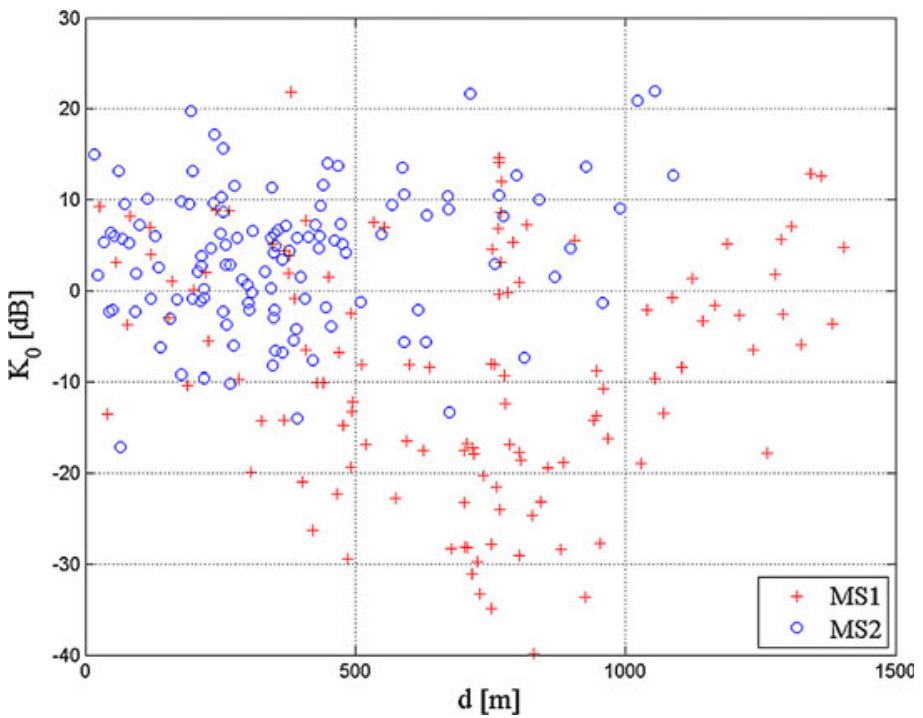

Fig. 14 Simulated channel $K_{0}$ factor for two mobiles in the Lisbon GBU environment

\subsection{Large-Scale Variations and Clustering}

An example of the dynamic evolution of simulated instantaneous power azimuth profiles for one of the MSs moving in the Lisbon GBU environment is shown in Figs. 15 and 16. A $5 \mathrm{MHz}$ band-limiting filter was applied to the modeled channel impulse response function.

The power azimuth profile (in $\mathrm{dB}$ ) at the BS (see Fig. 15) reveals a cluster appearance/disappearance, while the power azimuth profile at the MS (Fig. 16) shows an expected increased 


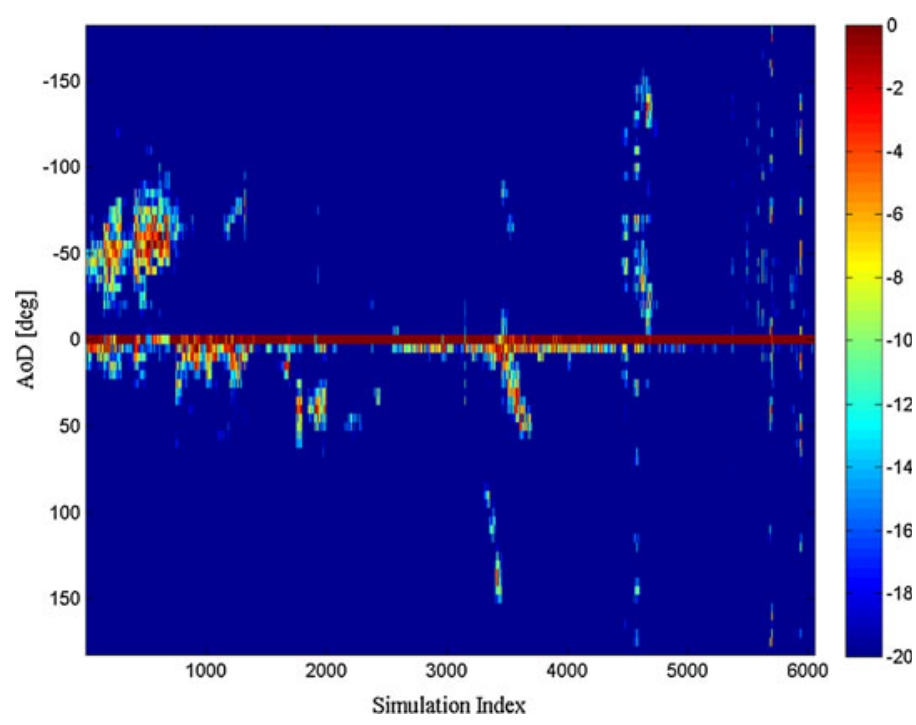

Fig. 15 Dynamic evolution of simulated power azimuth profile (AoD, at the BS) for a MS route in Lisbon using the extended COST 273 channel model

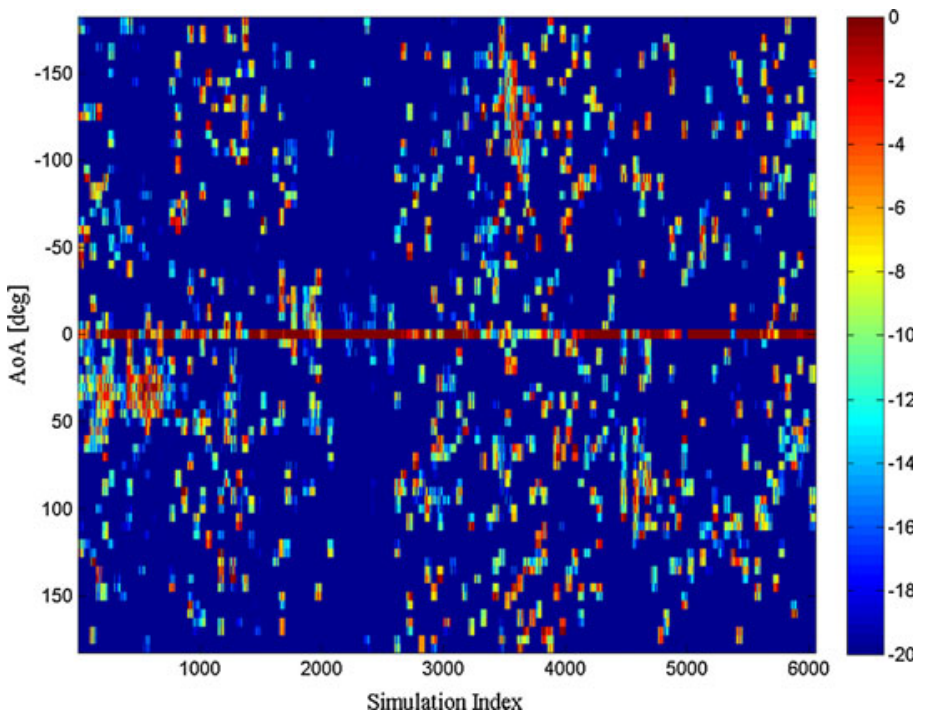

Fig. 16 Dynamic evolution of simulated power azimuth profile (AoA, at the MS) for a MS route in Lisbon using the extended COST 273 channel model

dispersion, caused by the MPC's arrival to the MS from almost all possible azimuths. This agrees with the macro-cell radio channel characteristics.

The simulation analysis was performed and delay/azimuth spread statistics were compared with other models and experimental data [13], revealing good agreement in the results. Additionally, the clutter and terrain effect produced an increase on channels delay and 
azimuth spread, compared with the original COST 273 DCM, which underlines Lisbon bad urban characteristics.

\section{Conclusions}

We have presented a new realistic DCM framework that is an extension of COST 273 channel model. The model uses a cluster and visibility generation based strategy with increased realism, due to the introduction of terrain and clutter information.

The model is based on a geometrical/stochastic interpretation, and allows a realistic treatment of both cluster positions and dynamics. This is done by means of visibility regions.

Using terrain and clutter information, the cluster and visibility region generating PDF were set.

New approaches for path-loss prediction were considered, affecting the cluster path gain model implementation. Additionally, it is introduced a new realistic approach for LoS stochastic modeling.

The new model was implemented in MATLAB ${ }^{\complement}$ using terrain, clutter, street and user mobility information for the city of Lisbon, Portugal. The simulation analysis was performed and results for path loss and small/large-scale fading were presented.

\section{References}

1. Hata, M. (1980). Empirical formula for propagation loss in land mobile radio services. IEEE Transactions on Vehicular Technology, 29, 317-325.

2. Comission of the European Communities. (1989). COST 207_digital land mobile radio communications. European Comission.

3. Correia, L. M. (Ed.). (2001). Wireless flexible personalised communications (COST 259 final report). Chichester, UK: John Wiley.

4. Correia, L. M. (Ed.). (2006). Mobile broadband multimedia networks, techniques, models and tools for 4G. GB: Academic Press, Elsevier Ltd.

5. Vieira, P., Vieira, M. A., Queluz, M. P., \& Rodrigues, A. (2005). A new realistic vehicular mobility model for wireless networks. In 8th International symposium of wireless personal multimedia communications 2005 (pp. 1805-1809). Alborg, Denmark.

6. Vieira, P., Vieira, M. A., Queluz, M. P., \& Rodrigues, A. (2006). Validation of a novel vehicular mobility model for wireless networks. In 9th International symposium of wireless personal multimedia communications 2006 (pp. 383-387). San Diego, USA.

7. Vieira, P., Queluz, M. P., \& Rodrigues, A. (2007). A dynamic propagation prediction platform over irregular terrain and buildings for wireless communications. In Proceedings of 66th IEEE vehicular technology conference fall 2007, Baltimore, USA.

8. Pedersen, K. I., Mogensen, P. E., \& Fleury, B. H. (1997). Power azimuth spectrum in outdoor environments. IEE Electronics Letters, 33(18), 1583-1584.

9. Adachi, F., Feeney, M. T., Williamson, A. G., \& Parsons, J. D. (1986). Crosscorrelation between the envelopes of $900 \mathrm{Mhz}$ signals received at a mobile radio base station site. IEE Proceedings, 133(6), 506-512.

10. Greenstein, L. J., Ergeg, V., Yeh, Y. S., \& Clark, M. V. (1997). A new path-gain/delayspread propagation model for digital cellular channels. IEEE Transactions on Vehicular Technology, 46, 477-485.

11. Vieira, P., Vieira, M. A., Queluz, M. P., \& Rodrigues, A. (2007). A novel vehicular mobility model for wireless networks. Wireless Personal Communications Journal Springer, 43, 1689-1703.

12. Vieira, P. (2007). Increasing realism in the cost 273 cluster path gain model. IT/IST internal report.

13. Vieira, P., Queluz, M. P., \& Rodrigues, A. (2007). Terrain and clutter impact on joint statistical properties of azimuth spread and delay spread in macro-cell bad urban environment. In Proceedings of IEEE international symposium on wireless communication systems 2007 (pp. 798-802). Trondheim, Norway. 


\section{Author Biographies}
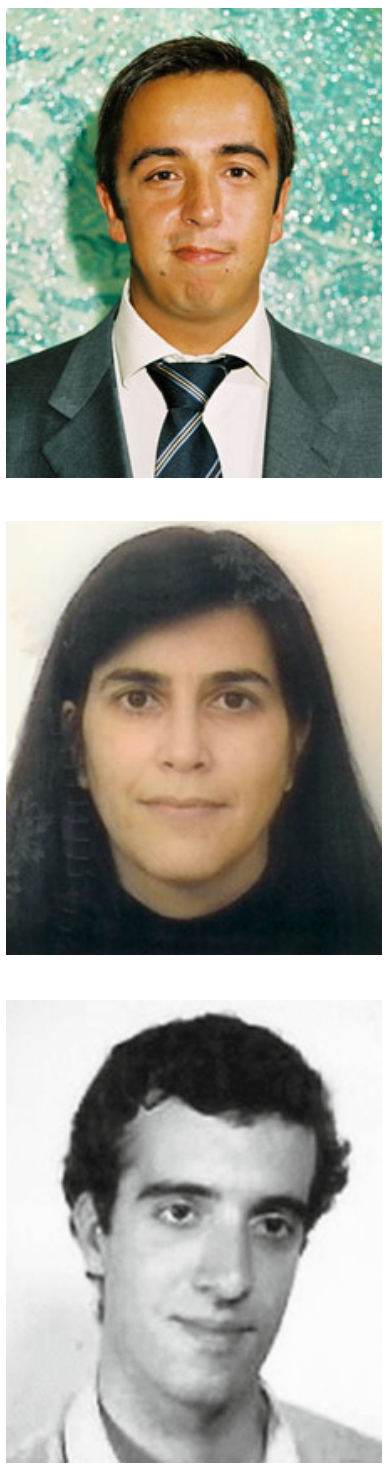

Pedro Vieira was born in Lisbon, Portugal, in 1974. He received the Engineering Degree in Electrical and Computer Engineering from Instituto Superior Técnico (IST) University, Lisbon, in 1997, and the M.S. and Ph.D. degree in Electrical and Computer Engineering from IST, Lisbon, in 2003 and 2008, respectively. He is currently a Professor in Lisbon Polytechnic Institute (ISEL), and a researcher at Instituto de Telecomunicações. His current interests include channel modeling, cellular mobile radio systems, digital radio transmission and MIMO systems.

Paula Queluz received the E.E. and the M.Sc. degrees in Electrical and Computer Engineering from the Instituto Superior Técnico, Technical University of Lisbon, Portugal, in 1985 and 1989 respectively, and the Ph.D. degree in Applied Sciences from the Catholic University of Leuven, Belgium, in 1996. She is presently Assistant Professor at the Technical University of Lisbon and researcher at Instituto de Telecomunicações, Lisbon. Her scientific interests include image analysis/processing, copyright protection and mobile communications.

António Rodrigues received the B.S. and M.S degrees in Electrical and Computer Engineering from the Instituto Superior Técnico (IST), Technical University of Lisbon, Lisbon, Portugal, in 1985 and 1989, respectively, and the $\mathrm{Ph}$.D. degree from the Catholic University of Louvain, Louvain-la-Neuve, Belgium, in 1997. Since 1985, he has been with the Department of Electrical and Computer Engineering, IST, where is currently an Assistant Professor. His current interests include modulation, coding, multiple access techniques, cellular mobile radio systems, digital radio transmission and diversity reception and equalization problems for mobile environments including satellite/mobile systems. 